\title{
Références bibliographiques du dossier «Confiance, éducation et autorité »
}

Bernadette Plumelle

\section{OpenEdition}

1 Journals

Édition électronique

URL : https://journals.openedition.org/ries/5544

DOI : $10.4000 /$ ries. 5544

ISSN : 2261-4265

Éditeur

France Education international

\section{Édition imprimée}

Date de publication : 1 septembre 2016

Pagination : 159-168

ISBN : 978-2-85420-611-1

ISSN : 1254-4590

Référence électronique

Bernadette Plumelle, "Références bibliographiques du dossier " Confiance, éducation et autorité » », Revue internationale d'éducation de Sèvres [En ligne], 72 I septembre 2016, mis en ligne le 01 septembre 2016, consulté le 02 juillet 2021. URL : http://journals.openedition.org/ries/5544 ; DOI : https://doi.org/ $10.4000 /$ ries.5544 


\title{
Références bibliographiques du dossier « Confiance, éducation et autorité "
}

\author{
Bernadette Plumelle \\ Centre de ressources et d'ingénierie \\ documentaires (CRID), CIEP
}

Les médias se font souvent l'écho de la question de la confiance au sein de l'école et de sa détérioration entre les différents acteurs - élèves, parents, enseignants, autorités scolaires. Les économistes et les sociologues ont largement étudié ce que pouvait représenter la confiance dans la vie économique et au sein des sociétés. L'importance de la confiance dans l'école a été plus tardivement étudiée. Par ailleurs, les travaux de sociologues et d'économistes qui montrent l'importance de la confiance entre les différents partenaires de l'école et l'effet qu'elle peut avoir sur l'amélioration de l'école et les résultats des élèves sont très majoritairement issus du monde anglo-saxon. La question de la confiance à l'école est rarement traitée en tant que telle dans les publications francophones.

Cette sélection bibliographique a été organisée en quatre parties. La première propose des références sur les notions d'autorité et de confiance hors du champ éducatif. Les auteurs appartiennent à différents champs des sciences sociales : philosophie, sociologie, économie, etc. La deuxième partie recense des publications sur les modèles éducatifs en débat actuellement. Les deux parties suivantes sont centrées sur le lien entre confiance et éducation. Les publications de la troisième partie ont pour objectif de cerner les relations de causalité entre la confiance et le développement de la scolarité ou montrent, de manière indirecte, les effets des politiques menées (reddition des comptes, autonomie des établissements scolaires, évaluations) sur le climat de confiance des acteurs du monde scolaire. On y constatera de nombreuses publications de l'OCDE sur ce sujet. Les références de la dernière partie sont des études des effets de la confiance / défiance dans différents contextes géographiques.

Cette sélection bibliographique n'a pas de prétention à l'exhaustivité et le choix a été fait de sélectionner des références récentes. De plus, les références proposées complètent celles qui sont fournies par les auteurs du dossier et qui ne sont pas reprises ici. Les résumés sont, pour l'essentiel, ceux des éditeurs ou des revues.

Bibliographie arrêtée le 26 août 2016.

\section{Les notions de confiance et d'autorité}

\begin{abstract}
CORNU Laurence, "La confiance », Le Télémaque 2/2003 ( $n^{\circ}$ 24), p. 21-30 [en ligne] [www.cairn.info/revue-le-telemaque-2003-2-page-21.htm]

La confiance est un fait humain, et certaines de ses formes sont des relations et des actes où se joue une émancipation. Elle ne peut être l'objet ni d'un impératif moral, ni d'un impératif technique. Mais on en fait l'expérience et on peut chercher à penser son effectivité paradoxale dans plusieurs approches (sociologique, éthico-politique, anthropologique, épistémologique), car elle est à l'œuvre, tout comme la méfiance, dans le lien social et transgénérationnel. Nous conduisant à interroger le statut de l'imprévisibilité dans nos pratiques et nos idéaux, cette notion peut inspirer des réflexions décisives dans l'éducation et la formation des maitres.
\end{abstract}


CORNU Laurence, "La confiance comme relation émancipatrice ", in OGIEN Albert, QUÉRÉ Louis (sous la direction de), Les moments de la confiance : connaissance, affects et engagements, Économica/Paris, 2006, p. 169-190

«La confiance, qui intéresse bien des champs de l'existence humaine, invite donc, pour pouvoir être comprise, à réfléchir également sur nos manières de saisir, sur leur paradigme "cognitif" et leurs visées pratiques ». L'auteure considère certaines formes de confiance comme des relations émancipatrices telle que la « confiance-abandon » de l'enfant à l'adulte. Elle propose trois axes théoriques - philosophique, anthropologique et éthico-politique - pour nourrir la réflexion autour de la notion de confiance.

ÉLOI Laurent, "Peut-on se fier à la confiance? ", Revue de l'OFCE, janvier 2009, $n^{\circ} 108$, p. 5-30 [en ligne] [http://goo.gl/x6D282]

Cet article propose une revue de la littérature de la recherche contemporaine sur le thème de la confiance, en tentant de circonscrire les rapports qu'elle entretient avec la théorie économique. Après avoir tenté d'éclairer le lien entre confiance interpersonnelle et intérêts personnels, il passe en revue différentes conceptions de la confiance - limitée, généralisée, confiance dans les institutions. La typologie des approches actuelles de ce concept et les différentes évaluations disponibles pour mesurer son niveau dans les sociétés contemporaines sont présentées et discutées.

LE BOUTER Flavien, "Formes et fonctions de la confiance dans la société moderne », Implications philosophiques, juin 2014, n. p. [en ligne] [http://goo. gl/jiLgde]

L'intérêt de la sociologie pour le thème de la confiance s'est considérablement accru ces dernières années, en particulier dans les pays anglo-saxons mais aussi en Allemagne. L'auteur cherche à clarifier les problèmes sociologiques que pose la confiance. Il s'attache à montrer que le passage à la société moderne s'est accompagné d'une transformation radicale du statut de la confiance : l'ordre social ne repose plus seulement sur la familiarité et la confiance personnelle mais aussi sur la confiance en des systèmes abstraits.

\section{LUHMANN Niklas, La confiance, un mécanisme de réduction de la complexité sociale, Économica/Paris, 2006, 123 p.}

Selon l'auteur, «la confiance au sens le plus large du terme, c'est-à-dire le fait de se fier à ses propres attentes, constitue une donnée élémentaire de la vie en société. Certes, l'homme a, en de nombreuses situations, le choix d'accorder ou non sa confiance à divers égards. Mais, s'il ne faisait pas confiance de manière courante, il n'arriverait même pas à quitter son lit le matin ». Sa définition de la confiance distingue une forme de confiance latente dans les institutions de la confiance accordée à tel individu ou entité. Il considère la confiance comme un outil qui a pour effet de réduire la complexité sociale et il fournit des outils de compréhension des interactions sociales et de la coopération entre individus.

\section{MENDEL Gérard, Une histoire de l'autorité, La Découverte/Paris, 2006, 294 p.}

L'autorité est en crise : ce constat, qu'il concerne la famille, l'école, l'entreprise ou l'État, relève aujourd'hui du sens commun. Mais qu'est-ce que l'autorité ? L'auteur met en évidence, de l'Inde à l'Afrique, de l'Antiquité aux temps modernes, un socle anthropologique commun : pour prendre en charge les peurs primaires de la vie, chaque société a interposé, lors des socialisations de l'enfance, la médiation d'une autorité protectrice, au prix de l'obéissance volontaire. Mais les formes de cette médiation ont beaucoup varié : dans les sociétés traditionnelles, la communauté exerce une autorité de type parental; dans les sociétés des temps modernes, c'est la figure du père qui incarne l'autorité. Aujourd'hui, en Occident, à l'heure du déclin de la société patriarcale, ni la communauté ni le père ne permettent plus d'apprivoiser suffisamment nos peurs archaïques, et c'est l'une des raisons profondes de la crise de l'autorité. 
OGIEN Albert, QUÉRÉ Louis (sous la direction de), Les moments de la confiance : connaissance, affects et engagements, Économica/Paris, 2006, 232 p.

La confiance est un phénomène social sans lequel nous ne pourrions ni agir ni interagir. Ce jugement n'a pas été démenti par les sciences sociales contemporaines, qui, à la suite de Luhmann ou de Giddens, considèrent la confiance comme un mécanisme de réduction du risque et de la complexité, qui instille un sentiment de sécurité propice au développement de la sociabilité. Les sciences politiques reprennent également cette définition : la confiance y est à la fois considérée comme une des conditions d'émergence de la démocratie et comme un facteur crucial à son fonctionnement, même si certains nient que la confiance soit un élément d'explication pertinent en politique. La confiance apparaît ainsi comme un rouage essentiel aux sociétés modernes, dont certains pensent même qu'il est devenu indispensable, à une époque où se multiplient les modes formels de régulation des transactions sociales (lois, codes, règlements, protocoles, normes, classements, etc.).

RENAUT Alain, La fin de l'autorité, Flammarion/Paris, 2004, 266 p.

Fragilisation de l'école, montée de l'individualisme, expérience toujours plus problématique de la parentalité : jamais les modèles d'après lesquels les adultes exercent leurs responsabilités à l'égard des enfants ne sont apparus aussi énigmatiques. Ni la posture réactive - il faut réarmer les formes anciennes de l'autorité - ni le discours progressiste - on ne doit à aucun prix brader les libertés acquises - ne s'attaquent frontalement à la question décisive : les formes de pouvoir qui, dans les sociétés traditionnelles, ont fonctionné sur le mode de l'autorité (éducative, mais aussi politique, judiciaire, médicale) sont-elles encore compatibles avec la logique de la démocratie?

TOMASELLO Michael, Pourquoi nous coopérons, Presses universitaires de Rennes/ Rennes, 2015, 85 p.

Grâce à l'observation de jeunes enfants, l'auteur, co-directeur de l'Institut Max-Planck pour l'anthropologie évolutionniste, montre que les enfants sont naturellement - et uniquement coopérateurs. Il identifie les processus psychologiques qui ont permis les premières formes humaines de coopération, jusqu'à nos formes uniques d'organisation culturelle, de l'évolution de la tolérance et de la confiance à la création de structures collectives (normes, institutions).

\section{Modèles éducatifs}

ALBERO Brigitte, GUEUDET Ghislaine, ENEAU Jérôme (sous la direction de), Formes d'éducation et processus d'émancipation, Presses universitaires de Rennes/ Rennes, 2015, 151 p.

L'ouvrage explore, selon une diversité de perspectives (philosophique, sociologique, didactique) et d'ancrages culturels les rapports contemporains entre éducation et émancipation. Le constat est fait des potentialités de l'éducation, en termes de contribution à l'émancipation et au processus démocratique. La première partie, de portée philosophique, analyse les phénomènes éducatifs liés aux mouvements de cultures en période de mondialisation. Les auteurs de la deuxième partie, sociologues, abordent la question de l'émancipation à partir de celle de la norme. La troisième partie souligne que la relation didactique impliquant un professeur et des élèves peut être émancipatrice, si elle aboutit au développement de l'élève.

\section{ARENDT Hannah, La crise de la culture : huit exercices de pensée politique, Gallimard/Paris, 1989, 380 p.}

L'ouvrage est un recueil d'articles extraits de diverses revues, dont deux textes ont pour sujet l'éducation. Dans le texte "Qu'est-ce que l'autorité » (1958), l'auteure définit l'autorité comme une forme d'obéissance qui ne requiert ni la persuasion ni la contrainte. Elle fait le constat que 
"l'autorité a disparu du monde moderne ». Le deuxième texte, "La crise de l'éducation », a pour terrain d'analyse la crise qui traverse la structure de l'organisation de l'éducation et de ses principes dans la société américaine. Hannah Arendt considère la crise de l'éducation comme une crise de l'autorité. L'école est ce qui permet la transition entre la famille et le monde; elle doit apprendre aux enfants ce qu'est le monde et non leur inculquer l'art d'y vivre. L'éducation sans enseignement n'est que rhétorique émotionnelle et morale.

\section{BLAIS Marie-Claude, GAUCHET Marcel, OTTAVI Dominique, "Fin ou métamor-} phose de l'autorité ", in Conditions de l'éducation, Stock/Paris, 2008, p. 135-212

Les auteurs proposent une réflexion sur ce qui fait problème aujourd'hui dans la transmission éducative. Toute une série de données qui servaient de socle à l'institution scolaire ont été ébranlées, voire sont en passe disparaître. Les auteurs mettent en lumière l'impact de ces transformations majeures et le défi qu'elles représentent. La troisième partie est consacrée à l'examen de la notion d'autorité dans l'éducation, complété d'une « esquisse d'une histoire des critiques de l'autorité en éducation ».

\section{DEWEY John, ZASK Joëlle, Démocratie et éducation suivi de Expérience et éducation, Armand Colin/Paris, 2011, 518 p.}

Ce volume propose les deux textes de John Dewey sur l'éducation : son traité de pédagogie, Démocratie et éducation (1916), et Expérience et éducation (1938). Dewey place l'expérience au cœur de l'apprentissage et recentre la pédagogie sur l'individu plutôt que sur les savoirs : l'école doit répondre à la curiosité naturelle de l'enfant et lui apprendre à désirer et entreprendre ; les travaux manuels et la vie sociale prennent le pas sur les exercices imposés; la motivation et l'effort individuel peuvent se substituer à la discipline et au régime de la sanction. La finalité de l'école est d'accroître la capacité à agir et c'est en cela qu'elle participe à la démocratie.

LOMBARD Jean, Hannah Arendt : Éducation et modernité, L'Harmattan/Paris, 2003, $116 p$.

Dans les analyses qu'elle avait consacrées à la crise de l'école américaine dans les années soixante, Hannah Arendt avait diagnostiqué le caractère fatal de la subversion de l'éducation par la pédagogie et du transfert de l'autorité au monde enfant, qui ont gagné depuis tous les systèmes éducatifs occidentaux. En définissant l'appareil conceptuel qui permet de penser l'école d'aujourd'hui, marquée par l'univers de la consommation, les phénomènes de masse, la perversion de la culture par le loisir, Hannah Arendt a édifié la philosophie de l'éducation de la modernité.

\section{PRAIRAT Eirick, L'autorité éducative : déclin, érosion ou métamorphose, Presses universitaires de Nancy/Nancy, 2010, 159 p.}

L'autorité ne va plus de soi. L'une des confusions les plus communes au sujet de l'autorité consiste à l'identifier à une simple relation de pouvoir, sans voir le lien fondamental qu'elle entretient avec le temps. La première partie de l'ouvrage réinterroge ce lien que la modernité semble distendre. Dans la seconde partie, il est exclusivement question d'école et d'enseignement. Si effectivement, l'autorité est intrinsèquement liée à la question de la transmission, alors l'école est bien le lieu «naturel » de l'autorité, le lieu où se joue le legs d'un héritage et où se met en scène le scénario de la crise. Est alors examinée une série de questions vives : l'héritage de l'éducation nouvelle, la question du partage de l'autorité entre parents et enseignants ou encore la façon dont se construit dans le jeu complexe des interactions, au sein même de la classe, une posture d'autorité.

\section{ROUILLER Yviane, LEHRAUS Katia (ed.), Vers des apprentissages en coopération : rencontres et perspectives, Berne: Peter Lang, 2008, 237 p.}

L'ouvrage regroupe des contributions offrant une pluralité d'éléments pour prendre conscience des enjeux de la coopération, de ses caractéristiques, de ses exigences, de ses variations et de ses influences. Il permet de mieux comprendre les similitudes et les différences entre des 
approches venant de l'Europe francophone et celles issues d'Amérique du Nord. Après une introduction qui rappelle les deux ancrages théoriques fondateurs des approches coopératives (les travaux de Piaget et ceux de Vygotski), deux grandes parties offrent un panorama de recherches et de réflexions. L'ouvrage se termine par un essai de "modélisation des conditions favorables aux apprentissages coopératifs », insistant sur la nécessaire alliance entre le social, le cognitif et l'affectif dans la coopération.

\title{
Éducation et confiance
}

\author{
ALGAN Yann, CAHUC Pierre, SHLEIFER Andrei, "Teaching Practices and Social \\ Capital ", Miméo Harvard, novembre 2010, 53 p. [en ligne] [http://goo.gl/2rukhN]
}

Le capital social est considéré comme la capacité des personnes, au sein d'une communauté, à coopérer avec les autres en dehors de leur famille. De nombreux chercheurs en sciences sociales font valoir que le capital social est persistant au fil du temps, en grande partie parce que les croyances sous-jacentes concernant les avantages de la confiance et de la coopération sont transmises dans les communautés par le biais des familles ou des interactions sociales. À partir de plusieurs sources de données internationales, les auteurs analysent en quoi le capital social est transmis ou non au sein de l'école à travers les pratiques d'enseignement. Au niveau individuel, les pratiques d'enseignement, verticales (relation frontale à l'enseignement, prise de notes du tableau) ou horizontales (travaux en groupe sur des projets) exercent une influence importante sur les croyances des élèves sur la confiance et la coopération, à la fois entre eux et avec les enseignants.

BJØRNSKOV Christian, Social trust and the growth of schooling, University of Aarhus - department of economics/Danemark, juin 2007, 52 p. [en ligne] [http:// goo.gl/cfEBEPQ]

L'auteur cherche à montrer que la confiance sociale exerce une influence forte sur le taux de croissance de la scolarité. Après avoir passé en revue les travaux qui ont cherché à déterminer le lien de causalité entre confiance sociale et scolarité dans un sens comme dans l'autre, l'auteur présente le lien théorique entre les deux et teste la validité de cette affirmation sur des données réelles, un échantillon de 52 pays, en mesurant la croissance de la scolarité entre 1960 et 2000.

BORGONOVI Francesca, BURNS Tracey, «The educational roots of trust ", OECD Education working papers, 2015, $n^{\circ} 119,56$ p. [en ligne] [http://goo.gl/H5gyJi] La confiance est un élément important du bien-être social et économique, qui permet de renforcer la cohésion sociale et la résilience, tout en maintenant la sécurité et l'ordre dans nos sociétés. Ces travaux ont pour objectif d'examiner le rapport entre l'éducation et les niveaux de confiance interpersonnelle, grâce aux données de l'étude de l'OCDE intitulée Programme pour l'évaluation internationale des adultes (PIAAC). Les auteures montrent que l'éducation renforce les capacités cognitives et analytiques nécessaires au développement, au maintien et (éventuellement) à la restauration de la confiance, à la fois dans les relations avec les proches et dans les relations avec le reste du monde. De ce fait, éducation et confiance sont intimement liées et interdépendantes.

BORGONOVI Francesca, ACHIRON Marilyn, L'égalité des sexes dans l'éducation : aptitudes, comportement et confiance, Paris : OCDE, 2015, 190 p. [en ligne] [http://goo.gl/Y9Y2cN]

L'ouvrage essaie de comprendre les raisons pour lesquelles, à l'âge de 15 ans, les garçons sont en moyenne plus susceptibles que les filles d'être peu performants dans toutes les matières, et les filles très performantes de réussir moins bien en mathématiques, en sciences et en résolution de problèmes que les garçons très performants. Selon les données du rapport, les différences 
de performance scolaire entre les sexes trouvent leur origine dans les attitudes des élèves à l'égard de l'apprentissage et leur comportement à l'école, dans la façon dont ils choisissent d'utiliser leur temps libre, et dans la confiance qu'ils ont - ou non - dans leurs propres capacités en tant qu'apprenants.

\section{BURNS Tracey, KOSTER Florian (ed.), Governing education in a complex world,} Paris : OCDE, avril 2016, 237 p. [en ligne] [http://goo.gl/Jdr7Ja]

Quels sont les modèles de gouvernance efficaces pour les systèmes éducatifs ? On observe une diversification et une complexité croissante des systèmes éducatifs. La première partie de l'ouvrage explore le concept de complexité et discute de ses implications pour la gouvernance des systèmes éducatifs. La deuxième partie se penche sur le rôle fondamental de la reddition de comptes, examinant les différents mécanismes et tendances en la matière. Les parties suivantes mettent l'accent sur le renforcement des capacités et l'utilisation des connaissances puis explorent différents outils pour des systèmes ouverts, dynamiques et stratégiques. Le dernier chapitre récapitule les enseignements tirés des travaux de l'OCDE, évoquant une question souvent négligée, selon les auteurs : une gouvernance réussie repose sur la confiance.

CERNA Lucie, "Trust: what it is and why it matters for governance and education ", OECD Education working papers, 2014, $n^{\circ} 108,67$ p. [en ligne] [http://goo.gl/b1LU13]

La confiance est un élément omniprésent dans l'élaboration et la mise en ouvre des mesures d'action publique dans de nombreux systèmes de gouvernance, $\mathrm{y}$ compris dans le domaine de l'éducation. Or la compréhension, la conceptualisation et l'évaluation de ces questions restent limitées. Qu'est-ce que la confiance et quelle est son importance pour la gouvernance, en particulier dans les systèmes d'éducation ? L'auteure examine les raisons pour lesquelles la confiance est primordiale pour l'élaboration des mesures d'action publique et à quel niveau elle s'inscrit dans les questions actuelles de gouvernance. Elle aborde les différentes définitions de la confiance et présente plusieurs manières de mesurer la confiance, tout en évoquant leurs points forts et leurs points faibles. Enfin, elle présente un simple modèle de confiance et de gouvernance et analyse la relation entre la confiance et les caractères propres aux systèmes éducatifs, comme la complexité, les asymétries d'information et de pouvoir, la collaboration/ coopération, le contrôle et l'obligation de rendre des comptes, la professionnalisation.

\section{MAROY Christian, "Accountability et confiance dans l'institution scolaire ", Éducation comparée, vol. 5, 2011, p. 127-154}

Dans quelle mesure la montée de la reddition de comptes et de l'évaluation en éducation estelle significative d'un déclin de la confiance de l'État, des administrateurs et des usagers, dans la capacité des organisations à remplir correctement leurs missions ? N'est-elle pas également significative d'une perte de confiance à l'égard des enseignants et de leur professionnalisme? L'article tente de répondre à ces questions à partir des théories de la confiance en sciences sociales. Selon l'auteur, les dispositifs d'accountability tendent surtout à réduire l'incertitude des clients/managers quant aux risques d'opportunisme et d'opacité des opérateurs scolaires.

\section{MYAMOTO Koji, HUERTA Maria del Carmen, KUBACKA Katarzyna et al., Skills for social progress: the power of social and emotional skills, OCDE/Paris, mars 2015, 140 p. [en ligne] [http://goo.gl/Rv2vct]}

Ce rapport de l'OCDE présente une synthèse des travaux destinés à identifier les compétences socio-affectives qui peuvent contribuer à améliorer les résultats futurs de l'enfant, en termes à la fois éducatifs, professionnels et sociaux. Les décideurs, les enseignants et les parents peuvent favoriser le développement de ces compétences en améliorant les environnements d'apprentissage au sein desquels se fait leur acquisition. L'analyse des données met en évidence le rôle important joué par les familles, l'école et les collectivités locales dans leur développement et la nécessité de garantir la cohérence entre les différents cadres d'apprentissage. 
TSCHANNEN-MORAN Megan, Trust matters: leadership for successful schools, San Francisco : Jossey-Bass, 2014, 336 p.

Ce livre est un guide à destination des enseignants pour mettre en place et maintenir la confiance au sein de leurs écoles et pour expliquer comment restaurer la confiance quand elle n'existe plus. Il décrit les cinq éléments clés sur lesquels les individus fondent leurs jugements de confiance (la bienveillance, l'honnêteté, la transparence, la fiabilité et la compétence) et explore les facteurs qui influent sur le développement de la confiance.

VAN MAELE Dimitri, FORSYTH Patrick B., VAN HOUTTE Mieke (eds), Trust and school Life: the role of trust for learning, teaching, leading, and bridging, Dordrecht: Springer, 2014, 352 p.

Ce livre collectif offre un panorama de la recherche sur la confiance dans l'éducation par des chercheurs de la nouvelle génération comme par des chercheurs chevronnés. Il propose une mise à jour de la recherche sur ce sujet et présente diverses orientations pour la recherche future : approches conceptuelles, innovations dans la mesure de la confiance, exploration des déterminants et des résultats de la confiance.

\title{
La question de la confiance dans les systèmes éducatifs : quelques exemples
}

\author{
ADAMS C. M., FORSYTH P. B. \& MITCHELL R. M., "The formation of Parent- \\ School Trust. A multilevel analysis », Educational Administration Quarterly, \\ 2009, vol. $45 n^{\circ} 1, p .4-33$
}

Les auteurs ont pour objectif d'expliciter les conditions de la confiance des parents envers l'école. L'étude menée aux États-Unis a été réalisée sur un échantillon de 79 écoles et 578 parents. Différentes mesures de la confiance ont été relevées, comme la confiance des parents à l'égard de l'école ou la confiance des enseignants à l'égard des parents et des élèves. Les auteurs montrent que la confiance exerce des effets directs plus forts sur les conditions d'efficacité d'enseignement et sur la motivation scolaire que sur les performances scolaires elles-mêmes. Ils font également la preuve que les chefs d'établissement peuvent construire et maintenir la confiance des parents, indépendamment du niveau socio-économique des parents, de la composition ethnique ou du niveau des élèves.

\section{ALGAN Yann, CAHUC Pierre, ZILBERBERG André, La fabrique de la défiance... et comment s'en sortir ?, Paris : Albin Michel, 2013, 188 p.}

Ce livre fait suite à un premier ouvrage publié en 2007 sur le même sujet. Les auteurs analysent la situation de défiance généralisée au cœur du mal-être français. Ils font état de recherches qui montrent les relations systématiques entre le fonctionnement de l'école, celui des entreprises, de l'État et l'harmonie sociale. Ils s'appuient sur des évaluations statistiques ou qualitatives menées de façon scientifique (les enquêtes Pisa sur l'école, par exemple) et considèrent que l'école est, entre autres, à la source de cette défiance généralisée : elle privilégie les meilleurs au lieu de jouer la coopération et fonctionne selon une organisation du travail de type hiérarchique.

AUDUC Jean-Louis, Parents et école : rétablir la confiance, Paris : Fondation Terra Nova, février 2011, 7 p. [en ligne] [http://goo.gl/JCTWIa]

En 2010, les sondages en France ont montré le développement d'un sentiment d'incompréhension grandissant des parents vis-à-vis du fonctionnement et des contenus d'enseignement. Les parents ont parfois la tentation de se tourner vers un enseignement privé qu'ils jugent porteur de continuité au niveau des valeurs, même s'il applique les mêmes réformes que le public. De même, le développement du secteur éducatif marchand est en large partie lié à l'angoisse des parents, le système scolaire leur semblant ne pas permettre la réussite de tous. 
L'auteur établit un diagnostic, affirmant que les relations entre les établissements et les familles sont parfois complexes et appelle à la nécessité d'un renouvellement des relations entre enseignants, établissements et parents d'élèves.

BOUVIER Alain, DUVAL Philippe (coordinateurs), "L'école et son contrôle : dossier ", Revue internationale d'éducation de Sèvres, septembre 2008, $n^{\circ} 48$, p. 27-152 [en ligne] [https://ries.revues.org/363]

Dans la crise de confiance que traversent aujourd'hui de nombreux systèmes éducatifs, l'éducation s'inscrit désormais dans des perspectives de régulations et dans des exigences de plus en plus fortes en matière de résultats. La notion de "contrôle ", considérée comme archaïque pendant plusieurs décennies, revient en force, sous des formes modernisées, en raison d'enjeux nouveaux, à la fois nationaux et mondiaux. On assiste en effet à une évolution de son rôle et à une redéfinition de son articulation avec les pratiques d' "évaluation ", qui se sont elles-mêmes considérablement développées depuis quinze ans.

BRAY Mark, "La confiance en jeu et la croissance de l'éducation de l'ombre. Études de cas : Inde, Malaisie, Taïwan ", Revue internationale d'éducation de Sèvres, avril 2015, $n^{\circ}$ 68, p. 81-92, [en ligne] [http://ries.revues.org/4356]

En Asie, l'éducation scolaire est un pilier des structures sociales des sociétés depuis des décennies, voire des siècles. Néanmoins, les décennies récentes ont vu se développer des signes de défiance des familles vis-à-vis de l'éducation scolaire, qui n'est plus perçue comme suffisante à elle seule pour répondre à leurs attentes. Une des manifestations les plus évidentes en est l'expansion d'un système d'« éducation de l'ombre », le soutien scolaire privé, qui prend des formes différentes selon les pays.

\section{BRYK Antony, SCHNEIDER Barbara, Trust in schools: A core resource for impro-} vement, New York: Russel Sage Foundation, 2002, 240 p.

Ce volume cherche à déterminer l'importance des relations sociales dans la mise en œuvre réussie de la réforme scolaire aux États-Unis. Il présente une étude menée dans 12 écoles élémentaires de Chicago durant trois ans, chaque école ayant fait l'objet d'une réorganisation en réponse à la Loi de Chicago School Reform de 1988, qui a appelé à une plus grande implication des parents et des leaders communautaires locaux dans leurs écoles de quartier. Les résultats montrent que les relations de confiance entre les différents acteurs : enseignants, parents, élèves, chefs d'établissement favorisent la réussite des élèves et rendent efficaces les efforts d'amélioration de l'école.

CARLESS David, "Trust, distrust and their impact on assessment reform ", Assessment and evaluation in higher education, vol. 34, $n^{\circ} 1, p .79-89$, [en ligne] [http://goo.gl/mb8CNp]

La vie universitaire est profondément affectée par la reddition des comptes et les audits de qualité. L'auteur s'appuie sur une étude de cas dans un établissement de formation des enseignants d'une université de Hong-Kong, pour montrer qu'un des facteurs qui entravent la mise en place des pratiques d'évaluation est le manque de confiance dans les dispositifs mis en place.

ELGOY Ingrid, HOMME Anne, "Individual or collective responses to accountability policies. A comparative study of teacher professionalism in Norway and Swede », Éducation comparée, vol. 5, p. 107-125

Cet article propose un examen des relations entre les politiques d'imputabilité et le professionnalisme enseignant dans deux pays européens : la Norvège et la Suède. Les politiques d'imputabilité sont ici approchées en tant qu'elles affectent tant les enseignants engagés localement et individuellement sur leur lieu de travail que la profession enseignante en tant que collectif participant aux décisions politiques. Le système éducatif suédois, au contraire du système éducatif norvégien, a évolué vers une large autonomie mais cette évolution n'est pas allée de pair avec la confiance. 
DE GRAUWE Anton, "La mise en œuvre de la décentralisation : des constats d'Asie et d'ailleurs ", Revue internationale d'éducation de Sèvres, juin 2014, n.p. [http://ries.revues.org/3791]

L'auteur présente les conclusions de recherches menées à l'IIPE-Unesco sur les politiques de décentralisation éducative dans les pays émergents et en voie de développement (Afrique de l'Ouest et de l'Est, Asie) au niveau local (écoles) et sur l'impact de la décentralisation sur les grands objectifs liés à l'accès, la qualité et l'équité. Une des difficultés des politiques de décentralisation concerne le choix entre l'autonomie laissée aux acteurs locaux et le cadre régulateur qui les guide et/ou contraint. Pour l'auteur «la principale raison de la tentation du niveau central de garder le contrôle est l'existence d'un doute sur la capacité des acteurs locaux, couplé à un manque de confiance dans les systèmes de contrôle ».

\section{FORSYTH Patrick B., ADAMS Curt M., HOY Wayne K. Collective trust: Why schools can't improve without it, New York: Teachers College press, Columbia University, 2011}

L'ouvrage offre de nouvelles perspectives et des connaissances pratiques sur la construction sociale de la confiance pour l'amélioration de l'école. Les auteurs font valoir que les écoles sont des organisations qui nécessitent des environnements caractérisés par des niveaux élevés de confiance collective pour être efficaces. L'ouvrage donne un aperçu historique de la notion de confiance et offre une revue de la recherche empirique exhaustive. Il présente un cadre théorique et un ensemble de mesures fiables et valides pour étudier la confiance collective ; il propose des lignes directrices pour la politique de l'éducation et la pratique du leadership et fournit aux praticiens des outils pour évaluer la culture de la confiance de leurs écoles.

HOPFENBECK Therese, TOLO Astrid, FLOREZ Teresa, EL MASRI Yasmine, Balancing trust and accountability? The assessment for learning programme in Norway, Paris : OCDE, septembre 2013, 100 p.

Cette étude porte sur la mise en œuvre de réformes éducatives en Norvège et plus précisément sur un nouveau système d'évaluation de la qualité des apprentissages (Vurdering for Loring) Le système éducatif norvégien est très décentralisé. Les autorités scolaires (comtés, municipalités, prestataires privés) sont responsables de la mise en œuvre de l'organisation des services scolaires et des activités. Le système éducatif et la profession enseignante bénéficient d'une reconnaissance relativement forte. Cette étude montre que la majorité des enseignants manifestent une certaine défiance vis-à-vis de cette nouvelle réforme de l'évaluation.

IKESAKO Hiroko, MIYAMOTO Koji, Fostering social and emotional skills through families schools and communities: summary of international evidence and implication for Japan's educational practices and research, Paris : OCDE, juin 2015, $n^{\circ} 121,76$ p., [en ligne] [http://goo.gl/3CXJNv]

Les compétences socio-affectives, telles que la persévérance, la sociabilité, l'estime de soi, la coopération, la confiance et l'empathie (vis-à-vis des autres) aident les individus à faire face aux défis du XXIe siècle et à tirer profit des opportunités qu'il offre. Les décideurs, les enseignants et les parents peuvent favoriser le développement de ces compétences en améliorant les environnements d'apprentissage au sein desquels se fait leur acquisition. Ce document examine les données internationales, notamment celles du Japon, afin de mieux identifier les cadres d'apprentissage susceptibles d'être propices à l'acquisition des compétences socio-affectives chez l'enfant. L'analyse des données, en provenance des États-Unis et du Royaume-Uni pour la plupart, met en évidence le rôle important que peuvent jouer les familles, l'école et la collectivité dans le développement des compétences socio-affectives chez l'enfant, et la nécessité de garantir la cohérence entre les différents cadres d'apprentissage. 
JELLAB Aziz, VELTCHEFF Caroline, VIN-DATICHE Didier, L'évaluation en collège et lycée : confiance et engagement des acteurs, Paris : Berger-Levrault, 2014, 272 p.

En France, la loi de refondation de l'école du 8 juillet 2013 considère l'amélioration du climat scolaire comme un objectif, donnant une importance plus grande aux conditions d'apprentissage et par voie de conséquence aux modalités d'évaluation. L'ouvrage cherche à identifier les valeurs (équité, égalité des chances, justice sociale...), les limites et les attentes qui accompagnent l'évaluation, en distinguant trois niveaux : l'évaluation en classe, l'évaluation du travail des enseignants, l'évaluation des unités d'enseignement. Les auteurs redéfinissent l'évaluation en la distinguant de la sanction, du contrôle et proposent des stratégies d'évaluation fondées sur la coopération entre acteurs, entre élèves, sur la mobilisation et la responsabilisation des acteurs (auto-évaluation...).

\section{MAULINI Olivier, PROGIN Laetitia (coordinateurs), Des établissements scolaires autonomes ? Entre inventivité des acteurs et éclatement du système, Paris : ESF, 2016, 192 p.}

Faut-il rendre les établissements scolaires autonomes ? Le débat est vif et les positions passionnées : décentraliser serait-il le remède idéal ou, au contraire, la porte ouverte au chacun pour soi et à la compétition généralisée ? Dans ce contexte, enseignants, autorités scolaires, parents d'élèves militent pour, à la fois, davantage de liberté et de règles, de confiance et de contrôle, de participation et de directives, de délégation et d'autorité... Cet ouvrage dresse un bilan prospectif et comparatif de la situation en France, en Belgique, en Suisse et au Québec. Une première partie montre comment la question de l'autonomie des établissements est revisitée par des politiques éducatives désormais en quête d'efficacité, de traçabilité et de légitimité. Une deuxième partie s'approche du terrain pour observer qui décide - et comment - lorsqu'un établissement gagne en marges de manœuvre et/ou en espaces de participation. La troisième partie descend au niveau de chaque acteur, de l'enseignant au ministre, pour mieux mesurer si l'autonomie reste aujourd'hui vécue par eux comme une conquête, comme un faux-semblant ou, éventuellement, comme un cadeau empoisonné.

MONS Nathalie, "Autonomie et contrôle du travail enseignant : une enquête dans les pays européens ", Revue internationale d'éducation de Sèvres, septembre 2008, $n^{\circ} 48$, p. 109-117, [en ligne] [https://ries.revues.org/449]

S'appuyant sur une enquête menée par l'agence Eurydice en 2008 auprès de vingt-huit pays européens, l'article montre les évolutions en cours en matière d'évaluation des enseignants qui traduit une défiance accrue de l'institution vis-à-vis de leurs capacités. Une évolution se dessine dans la plupart des pays, qui va vers un accroissement du «contrôle » des enseignants et de leur travail dans quatre domaines : mise en place de "contrôles » (externes, autoévaluations, interne par le chef d'établissement, interne par les pairs ; ces dispositifs pouvant se superposer); un contrôle des processus qui laisse de plus en plus la place à une évaluation par les résultats des élèves ; une évaluation qui oscille entre l'évaluation collective des équipes pédagogiques et une évaluation individuelle des enseignants; l'absence d'incitations ou de récompenses.

PONCELET Débora, DIERENDONCK Christophe, MANCUS Giovanna, "La confiance, un présupposé aux relations école-famille au préscolaire ", Éducation et formation, $n^{\circ}$ e-304-01, mars 2016, p. 39-58 [en ligne] [http://goo.gl/bVXKXT]

Les représentations ou croyances des futurs enseignants constituent des éléments-clés dans la mise en place d'un partenariat école-famille. Dans une analyse des opinions et croyances des étudiants de bachelor en sciences de l'éducation au Grand-Duché du Luxembourg, les auteurs montrent que dans l'anticipation des opinions des parents et dans les attentes envers ces mêmes parents en matière d'engagement scolaire, les choses sont très tôt différenciées. Il semble que comprendre la nature et le rôle même de la confiance que les futurs enseignants peuvent avoir envers les relations école-famille constitue un élément de la relation école-famille. Les auteurs soulignent que la constitution de cette confiance constitue un "préalable» des partenariats construits entre parents et enseignants. 